\title{
Cambios esperados en las funciones administrativas después de la emergencia sanitaria: estudio en directivos de España y Colombia
}

\section{Marcela Navia-Núñez y Yanna Stefanu-Lidorikiotu}

\section{Infocaribe \\ Departamento de Economía y Empresa, Universidad de La Rioja}

La enfermedad COVID-19 ha generado una parálisis mundial. Una empresa colombiana y una española analizaron el caso de directivos en la etapa de confinamiento por medio de un estudio asincrónico con el método cualitativo Delphi. En el estudio, participaron encargados de recursos humanos, mercadeo o ambas áreas. Se evaluaron grandes empresas, pequeñas y medianas empresas (pymes), organizaciones sin fines de lucro y agremiaciones ubicadas en España y Colombia. Si bien el objetivo inicial fue estudiar los cambios esperados en las relaciones interpersonales, a medida que los participantes abrieron temas de debate, se abordaron las funciones administrativas, que es el foco del presente artículo. La función administrativa de planeación ha sido la más afectada por la pandemia. Se encontró que se han extendido otras formas de organización. Tal es el caso del teletrabajo, cuyas dificultades para la interacción y la manera de ejercer su control simultáneo preocupan a los directivos. Por ello, se debe trabajar en lazos de confianza y comunicación. Por otro lado, en el período de cuarentena, se aprendió a tomar decisiones más rápidamente. Con respecto a las expectativas futuras, se espera que los directivos muestren más empatía hacia las necesidades ajenas y que prevalezca la funcionalidad de ellos en el desempeño de las tareas administrativas.

Palabras clave: Funciones administrativas, COVID-19, teletrabajo, directivos, crisis

\section{Expected changes in administrative functions after health emergency: study among Spanish and Colom- bian managers}

COVID-19 caused a worldwide paralysis. An asynchronous study among executive officers was developed, using the qualitative Delphi method, during the confinement stage, by a couple of companies, one from Colombia, another from Spain. Participants were human resources and marketing managers (some of them working in both areas) of large companies, SMEs, non-profit organizations, and professional unions, located in both countries. A forecast of expected changes in interpersonal relationships was the main objective of the study; however, as participants broadened the debate, topics related to administrative functions emerged. This paper focuses in the latter. Administrative planning was the most affected function by the pandemic. Organizational arranges like telecommuting became more common, their interaction and control difficulties raise concerns among directors. Strengthening trust and communication bonds is important. Decision making process was faster during the quarantine period, it is a lesson learned by the participants. Increased empathy to the personal necessities and functionality prevalence in the performance of administrative tasks are both in the future expected by participants.

Keywords: Administrative functions, COVID-19, telecommuting, managers, crisis 


\section{Mudanças esperadas nas funçóes administrativas após emergências sanitárias: estudo entre gerentes da Espanha e Colômbia}

A doença por COVID-19 gerou uma paralisia econômica no mundo. Uma empresa da Colômbia e outra da Espanha, abordaram as diretrizes diretamente na etapa de confinamento usando de um estudo assíncrono com o método qualitativo Delphi. No estudo participaram os encarregados das áreas de recursos de humanos ou comercialização, ou ambas de pequenas, médias e grandes empresas, organizaçôes sem fins lucrativos e agremiaçóes dos diferentes países. O objetivo inicial foi estudar as mudanças nas relaçóes interpessoais. No entanto, na medida que os participantes abriram os temas de debate, surgiram aspectos sobre as funçóes administrativas que é o foco do presente artigo. A função administrativa de planejamento foi o setor mais afetado pela pandemia. Popularizam-se outras formas de organizaçáo, como por exemplo, o teletrabalho, contudo, as principais dificuldades foram a interaçáo e a forma de exercer o seu controle, $o$ que preocupa os diretivos, pelo qual deve-se trabalhar em laços de confiança e comunicação. No período da quarentena aprendeu-se tomar decisôes mais rapidamente. O futuro esperado dos diretivos é mais empatia com as necessidades dos outros e a prevalência da funcionalidade no desempenho das tarefas administrativas.

Palavras-chave: Funçóes administrativas, COVID-19, teletrabalho, gerentes, crise

\section{Introducción}

Estudiar las funciones administrativas se ha convertido en una de las principales tareas de la administración de empresas, pues en ellas se concentra mucho del trabajo de coordinadores y gerentes. Más allá de que existan sistemas informáticos especializados en la organización de tareas, es fundamental que el directivo asuma dicha labor y, por lo tanto, desarrolle esa habilidad. $\mathrm{El}$ análisis de las funciones administrativas puede ser complejo, pues, en ocasiones, se trata de una actividad que se ejerce a partir de la práctica, es decir, desde el ensayo y error. Ya que abordar el tema directamente puede ser difícil, en determinadas circunstancias, pueden surgir otras vías para estudiarlo.

El presente estudio utilizó la plataforma Messagenes. Tiene una estructura similar a Facebook o Linke$\mathrm{dIn}$, redes sociales que muchos conocen. Por ello, la facilidad para usar Messagenes permite que surjan nuevos temas de debate entre los propios usuarios. El objetivo del estudio, inicialmente, no implicaba abordar las funciones administrativas, sino las rela- ciones interpersonales. Sin embargo, a medida que fue desarrollándose la comunicación y el debate, surgieron ideas sobre cómo está cambiando el papel de los directivos en cuanto a funciones administrativas en momentos de la emergencia sanitaria. Asimismo, aparecieron diálogos en torno al futuro de los directivos, pues el método Delphi, que fue utilizado para este estudio, busca propiciar temas de prospectiva. El artículo muestra resultados en el marco de la investigación realizada en España y Colombia con directivos de entidades sin fines de lucro y empresariales. Para ello, se utilizó la metodología cualitativa. Durante todo el estudio, los habitantes de España y Colombia se encontraban en confinamiento obligatorio por la emergencia sanitaria causada por el COVID-19. Estas circunstancias permitían imaginar de manera más fácil el futuro después de la cuarentena.

En el estudio, se plantearon tres temas: relaciones personales dentro de la organización, relaciones con clientes y proveedores, e impacto social. A partir es estos, los participantes abrían discusiones. El último tema tuvo menos acogida que los dos primeros. 
Los tres objetivos iniciales fueron cubiertos y se tratan en otro artículo. Lo valioso del presente escrito es que se analiza cómo se evaluó de manera efectiva otro punto trascendental en la administración de empresas de manera indirecta, pues los participantes propusieron temas que los demás desarrollaron y los moderadores permitieron que el diálogo fluyera. $\mathrm{Al}$ profundizar en los temas iniciales concernientes a las relaciones interpersonales, el debate se fue conduciendo hacia las exigencias actuales de las funciones administrativas. Como no era el tema principal, los participantes expresaron de manera abierta sus preocupaciones alrededor de la situación inédita que supone el confinamiento mundial.

Como se ha mencionado, en este trabajo, se presenta una aproximación al pensamiento de directivos acerca de los cambios en las funciones administrativas durante el confinamiento. Concretamente, se abordan aspectos a considerar tras el fin del confinamiento, como son la planeación, el control, la organización, la coordinación y la dirección. Estos tópicos aparecieron de manera transversal al abordarse los tres temas iniciales del estudio. Con respecto a los aspectos aludidos, Dextre y Del Pozo (2012) explican que la coherencia en las diferentes funciones administrativas constituye una administración eficiente. La planeación establece los objetivos, para lo cual se deben reconocer las necesidades de la demanda y las ventajas a ofrecer; es decir, es una forma de tomar decisiones anticipadamente. La organización se define como las actividades requeridas para conseguir los objetivos; por ello, debe considerar factores como la ubicación, y la extensión para determinar funciones y procedimientos. La dirección establece políticas que ayuden a fijar las funciones, y debe guiar y motivar a los integrantes para conseguir los objetivos. El control busca asegurarse de que las actividades planeadas se hayan cumplido a cabalidad. En los siguientes párrafos, se desarrollan con más detalle estos conceptos.

\section{Conceptos}

\subsection{Necesidades}

A partir de las ideas de Hampton, Araya y Pedreros (2013) presentan el concepto de «motivación» como aquello que impulsa a que la gente se comporte de determinada manera; los motivos impulsan la búsqueda de satisfacción de las necesidades. Igualmente, siguiendo las propuestas de Ferrell y Hirt, y Robbins y Couler, cuando la necesidad queda satisfecha, se reduce el impulso que motivó la conducta (citados en Araya \& Pedreros, 2013). Además, los especialistas señalan que hay diferentes formas de abordar el tema. Por ejemplo, se pueden clasificar las necesidades en básicas y secundarias. En la primera categoría, se encontrarían aspectos como abrigo y alimentación. En la segunda categoría, estaría el ego. Siguiendo esa línea, Maslow, el teórico más reconocido del tema, planteó en 1943 cinco tipos de necesidades: fisiológicas, de seguridad, sociales, autoestima y autorrealización (citado en Araya \& Pedreros, 2013). En principio, primero tienen que satisfacerse las necesidades más básicas para luego seguir con las demás.

\subsection{Funciones administrativas}

El teórico líder en el tema de las funciones administrativas es Fayol, que señala que un plan facilita la utilización de los recursos y permite escoger los mejores métodos en función de los objetivos (citado en Voxted, 2017). En cuanto a organización, Fayol definió «organizar» como construir una estructura, tanto material como humana (citado en Voxted, 2017). Al respecto, Vega y Salaiza (2016), tras revisar autores como Heyderbrand, Clegg, Torrent y Ficapal, y Vega, afirman lo siguiente: 
Los nuevos enfoques de organización buscan dominar la incertidumbre, promover la innovación, reducir los costos, aumentar la flexibilidad, utilizar las tecnologías de información y de comunicación, crear un ambiente propicio para agilizar y darle la mayor rapidez a las respuestas para finalmente generar conocimiento de manera grupal (p. 125).

Específicamente, en relación a la forma de organización de teletrabajo, Martínez (2012) la define como la actividad remunerada que se hace fuera de la sede de la empresa. Asimismo, supone que el trabajador se mantenga en contacto con la compañía. Por lo general, se ejecuta en el domicilio del empleado ${ }^{1}$ y la comunicación se realiza por medio de una computadora. Entre las ventajas del teletrabajo, están la flexibilidad en el horario y la reducción en desplazamiento. Los trabajadores exitosos en esta modalidad generalmente son los responsables y autónomos. Martínez anota también que en Europa es más popular que en Latinoamérica, aunque en este último territorio está creciendo progresivamente.

Guzmán y Abreo (2017) comentan que el teletrabajo se remonta a la crisis del petróleo en Estados Unidos en 1970, que llevó a buscar el ahorro de energía. Así como Martínez, Guzmán y Abreo señalan que la modalidad es usada con mayor recurrencia en Europa, sobre todo en Dinamarca y Holanda. Anotan que en Colombia se tenía registro de 39.767 teletrabajadores en 2013; esta cifra es muy inferior a la reportada en Argentina, con 589.157 en 2007. Guzmán y Abreo realizaron un estudio en Bucaramanga (Colombia) en el sector de calzado, que tiene una presencia importante en esa ciudad. Encontraron que $97 \%$ de las empresas encuestadas no han implementado teletrabajo; 70\% consideran que, en caso de implementarse, el teletrabajo necesariamente debe combinarse con presencia en la empresa. Según los empresarios, entre las características requeridas por parte de los trabajadores, se encuentran la disciplina y el compromiso. Además, 79\% de los empresarios consideran que el trabajador debe dominar el uso de internet.

Por otro lado, Montealegre, Delgado y Cubillos (2017) definen la gerencia como una técnica que implica la realización de actividades para lograr objetivos. Esto requiere coordinar a los integrantes del equipo. Además, señalan que la coordinación supone pensar maneras de influir en las personas para lograr los resultados previstos. Por su parte, Fayol apunta que la actividad de coordinación se orienta a armonizar las actividades y esfuerzos (citado en Voxted, 2017).

Otra de las funciones administrativas es la dirección. Mintzberg, uno de los autores más destacados en administración de empresas, describe el rol del directivo a partir de una serie de actividades que inician con el desarrollo de las relaciones interpersonales (citado en De Oliveira, Escriváo, Seldo \& Ferraudo, 2015). Estas actividades dan acceso a información sobre los roles que permiten tomar decisiones y diseñar estrategias. Asimismo, Terry (1971) define la dirección como la acción o influencia que ejerce el líder para que las otras personas trabajen unidas con algún propósito.

En relación a la información anterior, Ulrich plantea una matriz en la que presenta un eje vertical que se organiza desde hoy en dirección al futuro (citado en Velásquez, 2019). En dicha matriz, el «hoy» consiste en lo operativo; y el «futuro», en lo estratégico. El segundo eje (horizontal) se plantea desde la orientación a procesos hasta a la orientación a las personas. A partir de esta matriz, se proponen cuatro roles: a)

1 El prefijo 'tele' significa 'lejos'. 
experto administrativo, orientado a los procesos y lo operativo, que ejerce funciones básicas; b) socio de trabajadores, afín a las personas y lo operativo, que apoya a los trabajadores; c) socio estratégico, enfocado en los procesos y la estrategia, con un estilo de dirección centrado en alcanzar los objetivos empresariales; y d) agente de cambio, a partir de los cuadrantes personas y estrategia, que impulsa la cultura del cambio.

Finalmente se encuentra la función de control. Dextre y Del Pozo (2012) señalan que el propósito del control es «alertar, detectar y orientar la corrección de las desviaciones de planes» (p. 72). Se trata de una acción vigilante que busca designar soluciones durante un período de tiempo determinado. Dependiendo del tiempo, se puede clasificar el control en previo, simultáneo o posterior. El control previo se realiza cuando se busca evitar errores. El control simultáneo concurre con la actividad. El control posterior se dirige a la actividad culminada -caso de un arqueo-, en el que se comprueba conformidad.

En cuanto a la aplicación, Montealegre et al. (2017) realizaron un estudio sobre empresas agroindustriales en Tolima (Colombia). Encontraron que la mayoría de directivos cuentan con una estructura de organización formal y muy pocos encuestados manifestaron convocar a los grupos de trabajo para discutir sobre la visión. En general, manifestaron sentirse orientados a la realización de la tarea.

\subsection{Crisis}

Enrique (2013) comenta que, cuando hay una crisis, se pone a prueba la capacidad de reacción de la empresa. Especialmente lo que atañe a sus estructuras y sistemas es un reto que afecta la imagen pública. Siguiendo esa línea, la autora, partiendo de las ideas de Losada, propone utilizar redes sociales en situaciones de crisis por la rapidez de difusión de información, porque no existen intermediarios y porque permiten recibir retroalimentación directa de los interlocutores. Por su parte, Colomés presenta etapas para el manejo de crisis: tranquilidad, crítica moderada, conflicto, crisis de imagen de marca y control posterior a la crisis (citado en Enrique, 2013). En caso de haber un problema de este tipo, se sugiere ser transparente ante los diferentes stakeholders. Asimismo, se recomienda tomar prevenciones, es decir, planificar posibles respuestas en función a diferentes escenarios cuando la situación lo permite.

\subsection{Cambio organizacional}

A partir de las ideas de Burke y Lines, Rodríguez y Mladinic (2016) definen el cambio organizacional "como un cambio deliberadamente planificado en la estructura formal, sistemas, procesos o dominio mercado-producto de una organización, dirigido a mejorar el logro de uno o más objetivos organizacionales» (p. 2). Por su parte, Collerette y Deliste definen el cambio organizacional resaltando el tiempo: «Es toda modificación observada en la cultura o la estructura de un sistema organizativo y que posee un carácter relativamente perdurable» (citado en Pérez, Vilariño y Ronda, 2017, p. 288). Por otro lado, Vall propone el cambio organizacional a partir de los resultados: «Es un proceso de la organización para comprender y mejorar cualquiera y todos los procesos justificativos que pueda desarrollar una organización para el desempeño de cualquier tarea y para el logro de cualquier objetivo» (citado en Pérez et al., 2017, p. 288). El concepto también es definido desde el punto de vista del empleado, como señala Achilles: «Es un proceso de cambios planeado, en sistemas socio-técnico abierto, tendiente a aumentar la eficacia y salud de la organización para asegurar el crecimiento mutuo de las empresas y sus empleados» (citado en Pérez et al., 2017, p. 289). Por último, Marín-Idárraga y 
Cuartas-Marín consideran el entorno: «La adaptación de las organizaciones a diversas transformaciones que pueden sufrir en su ambiente interno o en su entorno" (citado en Pérez et al. 2017, p. 289).

Sobre las expectativas en torno al cambio organizacional, Vroom señala que un individuo actuará de cierta manera por el resultado esperado y por las posibles consecuencias (citado en Renko, Galen \& Bullough, 2012). Sin embargo, las expectativas se ven marcadas por la experiencia directa e indirecta, y los cambios esperados pueden no darse. En relación a ello, Rodríguez y Mladinic (2016) realizaron un estudio con trabajadores chilenos del sector minero, en el que se determinó que la información suministrada debe ser consistente para que el trabajador tenga una actitud más receptiva hacia el cambio organizacional.

\section{Metodología}

Para el estudio, se utilizó el método Delphi, que es una forma de prospectiva realizada con expertos dentro de las metodologías cualitativas o subjetivas. Se define como «un proceso sistemático e iterativo encaminado a la obtención de las opiniones, y si es posible el consenso, de un grupo de expertos» (Landeta citado en Ortega, 2008, p. 32). Ortega (2008) comenta los pasos para llevarlo a cabo. En primer lugar, se define el problema, se forma un grupo de expertos y se diseña el cuestionario. Luego, se realiza la discusión a partir de preguntas por etapas y se da retroalimentación a los expertos para que puedan hacer ajustes al cuestionario en la siguiente etapa. Finalmente, se realiza el análisis de los resultados. Se suele aplicar a grupos pequeños (alrededor de treinta personas) divididos en subgrupos. Antes, se hacía con el grupo reunido de manera presencial. En la actualidad, se sigue realizando de esa manera o por la comunicación en línea. Astirraga (2003) señala que no es necesario a toda costa encontrar unanimidad en las opiniones de los participantes, pero sí debe haber puntos de convergencia. A partir de las ideas de Asselin y Harper, y Falzarano y Pinto Zipp, García y Lena (2018) explican que el método Delphi es muy útil cuando hay poca evidencia empírica previa. Ese era el caso del estudio realizado para el presente artículo, ya que antes no ha habido una pandemia de alcance mundial.

Esta investigación tuvo como grupo objetivo a informantes con las siguientes características: a) directivos o ejecutivos con poder de decisión o cargos con responsabilidad; b) pertenecientes a entidades sin fines de lucro o entidades privadas; c) en ejercicio de su profesión en España o Colombia. Se configuraron los siguientes grupos con veinticuatro expertos procedentes de Espańa y Colombia: a) directivos del área comercial o de recursos humanos de empresas grandes (siete personas); b) directivos del área comercial o de recursos humanos de empresas de tamańo medio y pequeño (ocho personas); c) directivos de entidades sin fines de lucro (cinco personas); d) directivos de asociaciones empresariales o de cámaras de comercio (cuatro personas).

En los grupos empresariales, se contó con participantes de diversos sectores económicos: de funerarias, construcción, textiles, tecnología de la información, climatización, transporte, banca, producción de alimentos, industria papelera, producción de acero, comercialización de muebles, ferretería, publicidad, logística y cosmética. En cuanto a organizaciones sin fines de lucro, se consideraron entidades que trabajan en los siguientes frentes: actividad cultural, apoyo psicológico, desarrollo comunitario y medio ambiente.

Las empresas organizadoras son Invenies de España e Infocaribe de Colombia. El trabajo de campo de realizó desde el 16 de abril hasta el 26 de mayo de 2020, 
es decir, tras la declaratoria de emergencia sanitaria en ambos países. Se realizaron tres rondas con preguntas abiertas, a partir de las cuales surgieron más preguntas abiertas de parte de los informantes o de los moderadores. Los participantes plasmaban por escrito sus opiniones a preguntas abiertas a manera de debate por medio de la modalidad asincrónica. Las preguntas iniciales de cada tema eran iguales para todos los grupos y luego se ajustaban según la dinámica de cada grupo. Al terminar el diálogo en torno a cada tema, se elaboró el mismo cuestionario estructurado para todos los grupos. También se hicieron devoluciones parciales. Se procuró que estas devoluciones funcionaran como temas detonantes, pero sin dar demasiados detalles para que los participantes tuvieran libertad de opinar. En algunos momentos, se comentaron temas abordados por otros grupos. En el proceso, los expertos opinaron sobre los comentarios de sus compañeros. Al final, se entregaron a los expertos conclusiones que reunían resultados de todos los grupos. En este documento, no se presentan resultados de los cuestionarios cerrados. Los tres temas de debate fueron relaciones interpersonales dentro de la organización, relaciones con clientes y proveedores, e impacto social. Más allá de estos aspectos, se trataron de manera espontánea temas relacionados a las funciones administrativas, que es lo que se aborda en este documento; es decir, las funciones administrativas fueron investigadas de manera indirecta. La plataforma utilizada para la recolección de datos fue Messagenes.

\section{Análisis de resultados}

\subsection{Dirección}

Uno de los puntos más destacados del estudio es la toma de decisiones. Los participantes consideran que esta tarea pudo realizarse de manera más ágil en el confinamiento por el COVID-19, debido a la presión de la situación. Asimismo, la situación excepcional ha llevado a que se trabaje de manera unida para que la empresa avance en el nuevo entorno.

Por otra parte, los directivos estiman que las empresas y trabajadores deben llegar a acuerdos más allá de lo estipulado por los gobiernos. Por ejemplo, plantean que deben proponer nuevas normas en el escenario de la pandemia. De acuerdo con los expertos de los cuatro grupos, debe haber disponibilidad, compromiso, disciplina, y recepción hacia la flexibilidad de empleados y empleadores para lograr adaptarse a la nueva situación. Asimismo, señalan que deben redactarse protocolos incluso sobre temas regulados por ley, como salarios; períodos de vacaciones; horarios; y modalidad de trabajo, en caso de haber teletrabajo, o una combinación de trabajo presencial y trabajo a distancia. Estos puntos deben ser diseñados por etapas y deben informarse a los empleados de manera adecuada. Una directora comercial de una empresa grande mencionó lo siguiente: «es muy importante que los acuerdos que se hagan se cumplan y con rapidez, los trabajadores debemos tener auto regulación [sic] para poder cumplir con los compromisos, y generar confianza entre las partes».

\subsection{Planeación}

Con respecto al tema de planeación, no surgieron muchas ideas a partir de la reflexión sobre los tres temas iniciales (relaciones interpersonales dentro de la organización, relaciones con clientes y proveedores, e impacto social), debido a que los participantes mencionaron que se presentaron reestructuraciones empresariales que supusieron despidos, o ajustes en procesos y cargos por haber cambiado a productos de mayor demanda en las circunstancias actuales. Asimismo, los proyectos planeados perdieron relevancia. En general, pensaron en el corto o mediano plazos para implementar los cambios 
que se requieren para la modalidad de teletrabajo. Un comentario que se presentó en varias organizaciones sin fines de lucro es que deben fortalecer las alianzas con otro tipo de entidades, como universidades, para poder sobrevivir: «tejer redes de colaboración es esencial para mitigar la crisis, y, en general, para ayudar a construir un mundo mejor, más justo».

\subsection{Organización}

En relación a la organización, el tema de teletrabajo surgió en los cuatro sectores en estudio. Se analizó de manera exhaustiva, pues despertó gran interés. Sobre todo, en el caso de empresas grandes, los expertos anotaron que se flexibilizaron las condiciones de ergonomía, iluminación, nivel de ruido y riesgos psicosociales que normalmente se tienen en las instalaciones de la compañía. Una participante comentó que a largo plazo el trabajo en casa no es sostenible por esos factores, teniendo en cuenta aspectos que van desde la prevención de enfermedades laborales hasta la inspección de los empleadores. Además, varios participantes de los segmentos empresariales mencionan que el teletrabajo siguió teniendo el mismo horario laboral. Sin embargo, señalaron que la jornada debería ser más corta para que pueda conciliarse con la atención de los hijos, que también se encuentran en confinamiento por el cierre de los colegios. En general, en el grupo de trabajadores de empresas grandes, varios participantes consideran que, en el mediano plazo, se llegará a realizar $50 \%$ de trabajo telemático y $50 \%$ de trabajo presencial. Esto se volvería un punto de inflexión (cambio de tendencia) y una fórmula perfecta. A continuación, se presentan los aspectos presentados por los participantes.

\section{Ventajas del teletrabajo}

a) Permite el aislamiento actualmente requerido $y$ que ha sido impuesto por la crisis sanitaria. b) Faculta mayor conciliación con la vida familiar.

c) Supone ahorro de tiempo en transporte.

d) Hace posible contratar personas ubicadas en ciudades diferentes a la de la sede de la empresa.

e) Permite que haya mayor eficiencia y una mejor focalización en objetivos por tener reuniones (virtuales) más cortas y centradas en temas importantes (siempre y cuando haya experiencia con la tecnología necesaria o se logre una adaptación rápida).

\section{Desventajas del teletrabajo}

a) Dificulta la comunicación natural entre seres humanos.

b) Se reducen las interacciones familiares, por medio de las cuales surgen ideas innovadoras. En ese sentido, la comunicación es más plana.

c) Obstaculiza el contacto visual y la expresión corporal. Según un participante de gran empresa: «aprendemos a 'leer' a nuestros compañeros a través del contacto personal, de sus expresiones, en la forma que reaccionan. Al perderse el contacto personal se pierde también la comunicación no verbal».

d) Se depende de la señal de internet, que puede tener muchas fallas.

e) Complica la coordinación entre diferentes áreas de la empresa.

f) Sobre todo, al inicio, a algunos empleados les puede ser difícil adaptarse. Además, se pueden sentir aislados y desvinculados.

g) Algunos empleados no cuentan con los espacios ni mobiliario adecuados en casa. Además, la dinámica familiar puede cambiar y pueden presentarse accidentes. 
h) Algunas empresas pretenden disponibilidad «24/7»; por ello, los empleados terminan trabajando más allá de su horario laboral. En general, como señalaron los participantes, muchos sienten «que se trabaja más desde casa».

i) Puede aumentar el nivel de estrés tanto en los empleados como en los directivos. En el primer caso, el estrés se debe a la incertidumbre del futuro y la confusión por su papel en la empresa; en el segundo caso, el estrés se manifiesta por la dificultad en asumir control en los procesos. En el caso específico del confinamiento, muchas empresas implementaron el teletrabajo sin políticas claras, capacitaciones o nuevos canales de comunicación.

\section{Posibles soluciones para implementar el teletrabajo}

a) Disponer de un servicio de resolución de problemas

b) Tener desde la empresa una actitud abierta

c) Mostrar responsabilidad y eficiencia desde la parte del empleado

d) Cambiar los procesos y procedimientos de la empresa para hacerlos más flexibles

e) Procurar un ambiente de trabajo adecuado en casa

f) Brindar apoyo emocional y de habilidades de organización a los empleados

g) Estructurar un plan de trabajo que permita responder por las labores de la empresa y considere tiempos para los quehaceres del hogar

h) Revisar cada puesto de trabajo para evaluar si se encuentra recargado de funciones o, por el contrario, si realiza tareas que no se requieren. Puede ser necesario vincular encargados de comunicaciones (y de captación de fondos, en el caso de entidades sin fines de lucro)

i) Diseñar espacios para realizar encuentros virtuales de distensión, además de los destinados a las reuniones de trabajo

j) Procurar combinar con jornadas presenciales

k) Ofrecer capacitación y entrenamiento en uso de tecnologías, como en plataformas de comunicación

Sobre la seguridad, los problemas y las soluciones del teletrabajo

Los participantes consideran que el teletrabajo requiere mucha seguridad en los sistemas informáticos. Esto puedo tratarse con a) el dominio de aspectos técnicos, como sistemas en la nube, una conexión remota por VPN (red privada), la adquisición de licencias y herramientas de colaboración en línea; y b) aspectos administrativos, como políticas de seguridad y un coordinador de sistemas informáticos interno o externo a la empresa. Además, las políticas de seguridad deben garantizar confidencialidad, integridad, disponibilidad y protección de datos. Para ello, se deben instalar sistemas de apoyo. Una persona del grupo de empresas grandes manifestó que el gobierno debe regular hasta qué punto el empleado puede ser vigilado por la empresa sin afectar su privacidad, dado que posiblemente se use software para control.

\subsection{Coordinación}

En las pymes se considera que, en las empresas de su tamaño, la comunicación interna puede realizarse por medios como el correo electrónico y teléfono. Por su parte, en las grandes empresas se requiere mayor tecnología; por ello, suele ser costosa. Sin embargo, esto también depende del sector económico al que pertenece la empresa, pues la pyme dedicada a tecnología 
de la información tendría que implementar alta tecnología por su propia naturaleza.

Más allá del medio, los expertos del área de recursos humanos consideran que la situación del confinamiento ha permitido tomar consciencia sobre aspectos relacionados al ambiente laboral. Entre ellos, se encuentra la necesidad de escuchar más a los trabajadores y buscar maneras de que los empleados se sientan más seguros con su papel en la empresa. Además, después de la emergencia sanitaria, será necesario implementar capacitaciones y seguimiento sobre cambios en el comportamiento del personal que ha traído la crisis del COVID-19. Los participantes de cargos de recursos humanos manifiestan mayor preocupación por el trabajo a distancia, pues consideran que su labor requiere de la relación personal. Directivos del área comercial consideran que es importante definir quién, cuándo y cómo se implementarán las comunicaciones. Es posible que se requiera de comunicación más frecuente por los constantes cambios que se han dado a raíz del COVID-19.

Una encargada de recursos humanos del grupo de pymes señaló que le inquieta cómo manejar la incertidumbre generada en los trabajadores, quienes piensan que serán despedidos por la situación económica o que pueden ser rechazados en la empresa si contraen el virus. Como la interrogante correspondiente se publicó en el grupo de grandes empresas, una integrante de ese segmento propuso caminos de comunicación interna entre el jefe y los colaboradores de su área. También comentó que en su empresa se hizo una reunión virtual masiva con todos los empleados y el presidente de la compañía para «que escucharan de primera mano en qué [situación] estamos y para dónde vamos, con oportunidad de hacer preguntas que fueron contestadas directamente por el Presidente $[s i c]$.$» . Asimismo, señaló que otro tema de$ comunicación interna entre jefe y empleados consiste en las disposiciones gubernamentales, así como en las expectativas que tiene el superior sobre lo que hace el empleado. En ese sentido, la información debe ser más fluida, pues ya no se dan los «informes de pasillo", por medio de los que los trabajadores se enteraban de muchos aspectos en la empresa. Por ello, la información puede ser difundida con comunicados y videos en vivo que permitan el diálogo entre jefes y el personal para también saber cómo se sienten los empleados y qué dificultades han tenido. De hecho, la integrante de la gran empresa comentó que en su institución se realizó una encuesta diariamente al personal de atención al público. Por medio de esta vía, se busca conocer aspectos como el estado de salud y dificultades que atraviesan. Por ejemplo, al enterarse de problemas asociados al transporte, se pudo suministrar rápidamente transporte privado a ese sector del personal. La situación del confinamiento ha exigido que los administradores apoyen a su equipo de trabajo en sus nuevos roles con mayor creatividad y cercanía. El pronóstico es que, al culminar el confinamiento, los aprendizajes que han surgido por la modalidad de teletrabajo persistan.

Con respecto a los clientes, los entrevistados consideran que usar varios canales de comunicación con ellos es importante. Entre estos, las redes sociales resultan vitales en las circunstancias de la crisis por la emergencia sanitaria. Según un participante del grupo pyme, «La diferencia fundamental será la forma de comunicarnos con nuestros clientes, incluso para los sectores menos avezados a usar las nuevas tecnologías y las redes sociales en sus planes de acción comercial».

\subsection{Control}

Desde el punto de vista de la empresa, una preocupación con respecto al teletrabajo es el control. Para ello, los expertos afirman que se deben mejorar los indicadores, se tiene que mantener la comunicación fluida y sencilla, y, sobre todo, se debe robustecer la confianza. 


\section{Discusión de resultados}

Como el estudio se realizó durante el confinamiento y la crisis económica generada por el propio confinamiento, los directivos participantes compartieron que ellos y los miembros de sus equipos de trabajo vieron afectadas sus necesidades básicas. De acuerdo a la pirámide de Maslow, individuos que hasta hace poco buscaban satisfacer necesidades de autorrealización volvieron a preocuparse por lo más esencial.

Varias de las tendencias que se aceleraron con la cuarentena, como tener mayor flexibilidad y agilidad en la toma de decisiones, son procesos que ya venían descubriéndose desde hace varios años. Por ejemplo, Vega y Salaiza (2016) hacen referencia a esas dos tendencias y a la utilización de tecnologías de la información. Por otra parte, lo planteado por Martínez (2012) se correspondió con las experiencias y predicciones de los participantes del estudio, pues consideraron que el teletrabajo permite tener flexibilidad de horario y exige responsabilidad. De igual forma, las conclusiones de Guzmán y Abreo (2017) son similares a los resultados del presente estudio, pues los autores hallaron que los empresarios colombianos estimaban que el teletrabajo necesariamente debía combinarse con la modalidad presencial, y que se requería disciplina por parte del trabajador y dominio del uso de internet.

En cuanto a coordinación, buscar formas de apoyar al equipo de trabajo con creatividad y cercanía es coherente con lo expresado por Montealegre, et al. (2017), que explican que los directivos deben buscar maneras de influir en los empleados para lograr resultados. Esto va de la mano con lo señalado por Fayol, que indica que la acción de coordinar se orienta a armonizar esfuerzos (citado en Voxted, 2017). Siguiendo esa línea, en el estudio se encontró que los directivos han intentado desarrollar más las relaciones interpersonales con su equipo y esperan que el trato continúe de esa manera cuando acabe la emergencia sanitaria del COVID-19. Esto es coherente con el rol del directivo descrito por Mintzberg (citado en De Oliveira et al., 2015). Esta idea se asemeja a la propuesta de Ulrich con respecto a que el directivo sea socio de los trabajadores (citado en Velásquez, 2019). Además, el directivo, debe inclinarse al cambio, lo que implica que brinde condiciones de estabilidad emocional que permitan tener la fortaleza para enfrentar los desafíos actuales sin abandonar los objetivos empresariales. De esa manera, se mezclan tres de los cuatro roles planteados por Ulrich: socio de los trabajadores, socio estratégico y agente de cambio, entre los que destaca la orientación a los trabajadores.

Ningún directivo participante manifestó convocar a su equipo de trabajo para dialogar sobre la visión empresarial, aspecto que fue encontrado en un estudio elaborado en Colombia por Montealegre et al. (2017). Por otra parte, los resultados de esta investigación coincidieron con aspectos mencionados por Dextre y Del Pozo (2012), pues los directivos consultados mostraron preocupación por la dificultad en ejercer control simultáneo con la tarea en caso de que el teletrabajo prevalezca en un futuro. Otro punto en que coincide el estudio con lo expresado por Dextre y Del Pozo es en la necesidad de establecer políticas ante la nueva realidad, es decir, convergen las funciones de control y dirección.

De acuerdo con los participantes, el confinamiento dificultó cumplir con los objetivos planeados antes por la empresa. Ya que los cambios se debieron a factores externos y no fueron planificados, sería difícil entenderlos a partir de la definición de «cambio organizacional» de Burke y Lines (citados en Rodríguez \& Miladinic, 2016). El pronóstico de los participantes es que los cambios perdurarán después del confinamiento. De acuerdo a Collerette y Deliste, los cambios 
deben ser perdurables para considerarlos como cambios organizacionales (citados en Pérez et al., 2017). En el caso concerniente a este estudio, los cambios se han visto motivados o acelerados por el entorno (la crisis de la pandemia), por lo que se corresponden a la noción de cambio señalada por Marín y Cuartas (citados en Pérez et al., 2017).

Por las circunstancias del confinamiento, se ha presentado una diferencia entre las expectativas de los trabajadores y los directivos en cuanto al alcance de objetivos, con lo que se sigue la línea de lo explicado por Vroom (citado en Renko et al., 2012). Ya que el trabajador había invertido una cantidad de energía significativa en las actividades laborales, esperaba que estas se vieran compensadas. Sin embargo, la empresa no ha podido cumplir, por lo que el trabajador se siente decepcionado. Entonces, pierde la esperanza de que la empresa cumpla en el futuro con sus compromisos, por lo que se le dificulta encontrar motivación para atender sus responsabilidades.

En el estudio, se presentó un debate, pues algunos consideraron que, ante la crisis, la reacción de un grupo de empresas consistió en realizar despidos; otros sintieron que las organizaciones se preocuparon por el bienestar de los empleados. De acuerdo con Achiles, el cambio debe partir del crecimiento mutuo empresa-trabajadores (citado en Pérez et al., 2017). En ese sentido, parece que en algunas empresas ha faltado consistencia en la información suministrada a los trabajadores; este es un aspecto importante encontrado en un estudio realizado en Chile por Rodríguez y Mladinic (2016), quienes mencionan que la consistencia permite que el trabajador tenga actitud receptiva. Siguiendo esa línea, los participantes plantearon espontáneamente que se requieren nuevos acuerdos entre la empresa y el trabajador. Con respecto al manejo de crisis, los participantes del estudio coinciden con Losada en que

las redes sociales son un medio de comunicación efectivo por su alcance y su rapidez de difusión (citado en Enrique, 2013).

El método Delphi resultó útil en este estudio, pues no había mucha evidencia empírica previa, dado que nunca había habido un confinamiento global. Esto se asemeja a la investigación de García y Lena (2018), que también resalta por abordar un caso reciente $y$ poco estudiado. El método fue aplicado en líneas generales siguiendo los pasos dados por Ortega (2008): se definió el problema, se formó un grupo de expertos, se diseñó el cuestionario, se realizó la discusión por etapas, se dio retroalimentación al grupo y se hicieron ajustes al cuestionario de la siguiente etapa. Así, los temas abiertos por los propios participantes dieron lugar a nuevos tópicos de discusión que se retomaron en los cuestionarios; y que permitieron encontrar un material destacado alrededor de las funciones administrativas, que no eran el tema central al comenzar la investigación. Esto evidencia la flexibilidad de la herramienta.

\section{Conclusiones}

En líneas generales, con respecto a los resultados en función a los países estudiados (Colombia y España), no se perciben grandes diferencias; es decir, las preocupaciones y tendencias esperadas son similares. Los términos más usados por los participantes fueron «flexibilidad», «comunicación» y «cambio». Estos se constituyen como elementos importantes a considerar en la evolución de las funciones administrativas en la etapa posterior a la emergencia sanitaria. Los directivos de empresas consultados buscan formas para adaptarse a la nueva situación. Por su parte, los miembros de organizaciones sin fines de lucro manifiestan mayor preocupación por el futuro de la economía, especialmente en el caso de los trabajadores. 
Debido a la emergencia sanitaria del COVID-19, personas que estaban en lo alto de la pirámide de Maslow, de pronto, se vieron afectadas en los escalones bajos. Asumiendo que muchos pueden solucionar el tema de la provisión de alimentos por su cuenta, las empresas deben procurar seguridad en el trabajo para que en un futuro cercano se pueda volver a pensar en necesidades de autorrealización, que es el nivel en el que se ubican muchos productos del mercado. Esto también es definitivo para el mercado interno, es decir, para los empleados. Bajo estas circunstancias, es muy importante que se genere confianza entre el empleador y los empleados, y entre los mismos empleados. Los jefes deben propender por fortalecer las habilidades de cada uno y tratar de conservar la tranquilidad. Como seres humanos, se debe mantener la seguridad física y emocional.

Durante el confinamiento, se han generado fricciones entre directivos y trabajadores: debido a los cambios constantes, se ven modificadas las reglas de juego. En algunas empresas, hay una tendencia a considerar que la situación radical de la pandemia permite romper los contratos sociales ya establecidos. Sin embargo, los trabajadores esperan estabilidad. En ese sentido, podrían entender nuevos acuerdos si son consultados al respecto. Por ello, la comunicación es vital: debe ser frecuente, clara y coherente. Las empresas y trabajadores deben llegar a acuerdos más allá de las nuevas normas establecidas por los gobiernos. Según los expertos de los cuatro grupos estudiados, debe haber disponibilidad, compromiso, disciplina y recepción hacia la flexibilidad de ambas partes para lograr adaptarse a la nueva situación. Por eso, deben redactarse protocolos en relación a temas que incluso han sido regulados por la ley, como salarios; períodos de vacaciones; horario; y modalidad de trabajo, en caso de haber teletrabajo o una combinación de este con trabajo pre- sencial. Estas decisiones deben plantearse por etapas e informarse a los empleados de manera adecuada.

Una de las ventajas observadas en el teletrabajo ha sido la mayor eficiencia y posibilidad de focalizarse en los objetivos. Estos aspectos se deben a que se realizan reuniones virtuales más cortas que en la modalidad presencial y se centran en temas importantes, siempre y cuando haya experiencia con respecto al uso de la tecnología necesaria o se logre una adaptación rápida. Como desventaja del teletrabajo, se observa que puede aumentar el nivel de estrés tanto en los empleados como en los directivos. Los primeros se verían afectados por la incertidumbre frente al futuro y la confusión sobre su papel en su posición laboral; los segundos se ven perjudicados por la dificultad en el control y el manejo de procesos.

En relación con la tecnología, el estudio ha mostrado una mirada dicotómica al respecto. Por un lado, se manifiesta un temor, pues puede reemplazar el trabajo de seres humanos. Por otro lado, se agradece la disposición de diferentes tecnologías, pues permiten solucionar problemas y estar en contacto con el mundo exterior en la situación de confinamiento. Sin embargo, las empresas medianas y pequeñas perciben que los cambios de transformación tecnológica son muy costosos frente a sus recursos disponibles. Para que el teletrabajo funcione en la etapa posterior al confinamiento, la capacitación sobre herramientas tecnológicas es necesaria, sobre todo, en el caso de trabajadores que no están acostumbrados a usarlas. Una alternativa para la capacitación puede ser la transmisión de videos que muestren cómo funcionan dichas herramientas. También es importante tener cubiertos los temas de seguridad de la información.

La función administrativa de planeación fue la que se vio más afectada por la pandemia, pues, por factores 
externos, muchos de los proyectos quedaron aplazados. Asimismo, se establecieron formas de organización alternativas, como el teletrabajo. Este medio permite continuar con las labores a pesar de la crisis sanitaria, pero preocupa a los directivos por las dificultades que supone para la interacción y la manera de ejercer control hacia su equipo de trabajo. Para confrontar esto, se deben establecer acuerdos y tiene que haber confianza mutua. Más que pensar en medidas fuertes de control simultáneo, se debe velar por una comunicación profunda y cercana a pesar de la distancia. Ante el temor de los empleados, es un reto para los directivos mantener la calma. Una ventaja de la situación es que se han tomado decisiones de manera más rápida, condición que probablemente se mantendrá a largo plazo. Esto se verá como una oportunidad para mejorar la efectividad, pues a veces en las empresas se tienen que hacer muchos trámites que retardan las acciones. Aun así, la realización de más acciones no debe implicar dejar de asumir rigurosidad y responsabilidad en el trato con los empleados, los clientes y la sociedad en general. Así, después de la pandemia, se espera que los directivos tengan mayor empatía frente a las necesidades ajenas, sin descuidar la funcionalidad en el desempeño de las funciones administrativas. Estas consideraciones deben ir acompañadas del uso asertivo de tecnología de información, que requiere de capacitación y un cambio organizacional a mediano plazo para enfrentar la crisis.

El uso del método Delphi permitió que hubiera flexibilidad para que los participantes del estudio propusieran tópicos y debatieran al respecto. Esto evidencia que el método permite dar lugar a nuevas ideas cuando no hay mucha información sobre un tema, como es el caso estudiado contextualizado en la crisis sanitaria del COVID-19, una parálisis mundial sin precedentes. El uso del método cuando hay poca información ya fue planteado anteriormente por
García y Lena (2018). Al utilizar el método Delphi, el moderador asume la función de motivación y su papel de organizador pasa a un segundo plano. También fue conveniente, pues permitió interactuar con pares de dos países, que pudieron transmitir ideas para solucionar problemas. A pesar de haber mucha información sobre el COVID-19 en los medios de comunicación y que el estudio hacía también presente el tema para los participantes, la intervención en la plataforma significó un respiro frente a las responsabilidades habituales y generaba curiosidad por las nuevas intervenciones que habría. Además, realizar el estudio de manera asincrónica permitió contrarrestar las dificultades en torno a la diferencia horaria de los dos países y las ocupaciones de cada participante.

En investigaciones futuras, se recomienda ahondar en alguna función administrativa específica, como la planeación o el control en situaciones de crisis. También se pueden involucrar a más países y se podría realizar una comparación con las percepciones de trabajadores de niveles más bajos en la organización. Como en el caso de esta investigación, se puede seguir explorando nuevos caminos con el uso del método Delphi de forma indirecta, que permitan profundizar en el papel de los participantes y del moderador.

\section{Referencias}

Araya, L. \& Pedreros, M. (2013). Análisis de las teorías de motivación de contenidouna aplicación al mercado laboral de Chile del año 2009. Revista de Ciencias Sociales, 4(142), 45-61. Recuperado de https://www. redalyc.org/articulo.oa?id=15333870004 [Consulta: 1 de julio de 2000].

Astirraga, E. (2003). El método Delphi. San Sebastián: Universidad de Deusto. Recuperado de http://www. prospectiva.eu/zaharra/Metodo_delphi.pdf [Consulta: 1 de junio de 2020].

Contabilidad y Negocios (15) 30, 2020 / ISSN 1992-1896 
De Oliveira, J., Escriváo, E., Seldo, M. \& Ferraudo, A. (2015). Managerial Styles of Small Business Owners: A Study Based on the Organizational Life Cycle and on Concepts Concerning Managers' Functions and Roles. Revista Brasileira de Gestão de Negócios, 17(57), 12791299. https://doi.org/10.7819/rbgn.v17i57.1650

Dextre, J. \& Del Pozo, R. (2012) ¿Control de gestión o gestión de control? Contabilidad y Negocios, 7(14), 69-80. Recuperado de http://revistas.pucp.edu.pe/ index.php/contabilidadyNegocios/article/view/3880 [Consulta: 30 de junio de 2020].

Enrique, A. (2013). La gestión de comunicación de crisis en las redes sociales. ORBIS, 24(8), 116-131. Recuperado de https://ddd.uab.cat/pub/artpub/2013/159701/ orbis_a2013v8n24p116.pdf [Consulta: 20 de junio de 2020].

García, M.E. \& Lena, F.J. (2018). Aplicación del método Delphi en el diseño de una investigación cuantitativa sobre el fenómeno FABLAB. Empiria. Revista de Metodología de Ciencias Sociales, 40, 129-166. https://doi. org/10.5944/empiria.40.2018.22014

Guzmán, A. \& Abreo, C. (2017). Las habilidades del teletrabajador para la competitividad. Forum Empresarial, 22(2), 5-30. https://doi.org/10.33801/fe.v22i2.13624

Martínez, R. (2012). El teletrabajo como tendencia del mercado laboral. RETOS. Revista de Ciencias de la Administración y Economía, 2(4), 143-156. https:// doi.org/10.17163/ret.n4.2012.01

Montealegre, J., Delgado, A., \& Cubillos, C. (2017). Relaciones entre modelos gerenciales y pensamiento estratégico empresarial en las empresas agroindustriales del departamento del Tolima. Pensamiento y Gestión, 42, 26-47. https://doi.org/10.14482/pege.41.9704

Ortega, F. (2008). El método Delphi, prospectiva en ciencias sociales a través del análisis de un caso práctico. Revista EAN, 64, 31-54. https://doi. org/10.21158/01208160.n64.2008.452

Pérez, L., Vilarińo, C. \& Ronda, G. (2017). El cambio organizacional como herramienta para coadyuvar la implementación de la estrategia. Ingeniería Industrial, 38(3), 286-294. Recuperado de http://rii.cujae.edu. cu/index.php/revistaind/article/view/605 [Consulta: 15 de junio de 2020].

Renko, M., Galen Kroeck, K. \& Bullough, A. (2012) Expectancy Theory and Nascent Entrepreneurship. Small Business Economics, 39, 667-684. https://doi. org/10.1007/s11187-011-9354-3

Rodríguez, V.,\& Mladinic, A. (2016). Ambivalencia actitudinal ante el cambio organizacional: un análisis desde el individuo en contexto laboral. Psykhe, 25(1), 1-17. https://doi.org/10.7764/psykhe.25.1.733

Terry, G. (1971). Principios de administración. México, D.F.: Continental.

Vega, L. \& Salaiza, F. (2016). Nuevas formas de organización para la innovación administrativa y técnica. Estudios Interdisciplinarios en Ciencias Sociales, 18(1), 127-137. Recuperado de https://www.redalyc.org/ articulo.oa?id=99344833008 [Consulta: 29 de junio de 2020].

Velásquez, A. (2019). Implementación del modelo de gestión humana propuesto por Dave Ulrich. Efectos, desafíos y percepciones (Tesis de maestría). Universidad EAFIT, Escuela de Administración. Medellín, Colombia. Recuperado de https://repository.eafit.edu. co/bitstream/handle/10784/13899/Andres_VelasquezGomez_2019.pdf? sequence $=2 \&$ isAllowed $=y$ [Consulta: 19 de agosto de 2020].

Voxted, S. (2017). 100 years of Henri Fayol. MREV Management Revue, 28(2), 256-274. https:/doi. org/10.5771/0935-9915-2017-2-256 


\section{Apéndices}

\section{Apéndice 1. Respuestas parciales para información de la revista ${ }^{23}$}

\section{Empresas grandes}

\section{Participante 1 (Colombia)}

\section{Nuevos acuerdos entre empresa y trabajador ante los cam- bios constantes}

Lo primero es la comunicación, que los trabajadores tengan claro los cambios que se están presentando y lo que se espera de él. Además pienso que los acuerdos se deben resumir en flexibilidad, por parte del trabajador porque seguramente tendrá que hacer cosas que no estaban acordadas antes, o trabajar en condiciones diferentes, y por parte de la empresa porque debe entender que los trabajadores necesitan adaptarse a los cambios y trabajar tal vez en horarios diferentes.

Es muy importante que los acuerdos que se hagan se cumplan y con rapidez, los trabajadores debemos tener auto regulación $[s i c]$ para poder cumplir con los compromisos, y generar CONFIANZA entre las partes.

Además de la comunicación interna entre jefe - colaborador, hicimos ya una primera gran reunión virtual del Presidente $[s i c]$ con todos los colaboradores de la compañía para que escucharan de primera mano en qué estamos y hacia donde [sic] vamos, con oportunidad de hacer preguntas que fueron contestadas directamente por el Presidente.

Cada vez cobra más importancia la comunicación interna, no solo por los cambios que todos los días se presentan no solo en las organizaciones sino en las reglamentaciones gubernamentales para prevenir la propagación del COVID-19, y por el hecho de estar separados ya ni siquiera se tienen los «informes de pasillo» que nunca faltan, de manera que cada lider [sic] es responsable por mantener a su equipo informado sobre el rumbo que está tomando la compañía y lo que se espera de él, también es importante que la alta dirección se deje «ver» a traves $[s i c]$ de videos, comunicados, o reuniones en vivo, y que la comunicación sea en doble vía no solo informar sino escuchar cómo se sienten, qué dificultades están teniendo. En la empresa donde yo trabajo se hace todos los días una encuesta a todo el personal que está atendiendo público donde preguntamos por su estado de salud, sus temores, sus dificultades para transportarse o en el trabajo, y sobre todos los días lo revisamos y se toman acciones correctivas si es necesario (p. ej. contratar un transporte privado para que no usen transporte público).

\section{Participante 2 (Colombia)}

\section{Comunicación interna}

Aprendemos a "leer" a nuestros compañeros a través del contacto personal, de sus expresiones, en la forma como reacciona. Al perderse el contacto personal, se pierde también la comunicación no verbal, aquella que te permite saber cómo estuvo la reunión de los directivos por la forma como entra tu jefe a la oficina una vez acabada la reunión (jajaja).

Se requiere aumentar la comunicación y definir los temas a hablar, es muy importante que durante la crisis y aun mucho después fluya la comunicación a lo largo y ancho de la empresa. Si los directivos no comunican

2 Las respuestas no pueden ser publicadas fuera de este artículo.

3 Se han trascrito de manera textual las respuestas ofrecidas por los participantes, es decir, se ha respetado la redacción original. 
las acciones, la incertidumbre se va a apoderar aun mas $[s i c]$ de los empleados y a generar mucha inestabilidad, desorientación y baja productividad. Se debe definir que se comunica, quien lo comunica y cuando, lo ideal es que sea en cascada y tan frecuente como sea necesario. En estos días de cuarentena podría ser incluso diario si los cambios al interior de la organización así lo son o semanales y mantenerlos así sea mensualmente hasta después de encontrada la cura y durante la recuperación de la economía.

\section{Organizaciones sin ánimo de lucro}

\section{Participante 3 (España)}

\section{Alianzas}

Siempre le hemos dado mucha importancia a las alianzas, tanto con otras organizaciones sociales como con empresas. De hecho, tenemos un «club» de empresas pioneras que son todas aquellas que nos financian o colaboran con nosotros de cualquier modo y están muy presentes en todas nuestras comunicaciones. En todo caso, creo que es un aspecto en el que se debería profundizar: tratar de que todas ellas interioricen a la entidad como su aliado principal en su política de RSC. Con la Universidad de La Rioja no solo tenemos relacion $[s i c]$ sino que forma parte de nuestro patronato. Pienso que tejer redes de colaboración es esencial para mitigar la crisis, y, en general, para ayudar a construir un mundo mejor, más justo.

\section{Pymes}

\section{Participante 4 (España)}

\section{Comunicación y coordinación}

Sin duda, habrá un antes y un después en las relaciones con clientes y proveedores. La diferencia fundamental será la forma de comunicarnos con nuestros clientes, incluso para los sectores menos avezados a usar las nuevas tecnologías y las redes sociales en sus planes de acción comercial.

Deberemos actualizarnos rápidamente, revisar el diseño de nuestras páginas web, desinvertir en ciertas acciones comerciales (grandes eventos por ejemplo) para invertir en otras más alineadas con el nuevo escenario post-covid.

Tendremos que adecuar también las estructuras de las empresas para dotarlas de músculo en el área de marketing y comunicación, fundamentalmente para la búsqueda de clientes nuevos, desarrollo de acciones específicas para prescriptores (en caso de que formen parte del negocio), etc.

La fuerza comercial se deberá reinventar y el vendedor deberá adquirir nuevos conocimientos a nivel de comunicación para usar adecuadamente las nuevas herramientas que se convertirán fundamentales para poder desarrollar la acción comercial.

En definitiva, en aquellos sectores con mayor déficit en el uso de nuevas tecnologías para el desarrollo de la acción comercial, la era post-covid supondrá un extraordinario impulso para modificar estrategias.

\section{Participante 5 (Colombia)}

\section{Seguridad informática en teletrabajo}

Una de las mayores preocupaciones es garantizar la confidencialidad, integridad y disponibilidad de los datos, la información es valiosa para todos y requiere de sistemas de protección. Cuando se habla de seguridad informática se debe considerar la seguridad de la información, para prevenir posibles ataques que provoquen la pérdida física o la modificación de la información que generamos; y algo bien importante como es la protección de datos, de las bases de datos y 
particularmente de datos sensibles en nuestras empresas. Entre los temores que se tienen en el momento de adoptar teletrabajo está el poner en riesgo la información, sé que es uno de los temas cruciales en esta situación que nos ha tocado, pues se siente como un mayor riesgo de que $[s i c]$ seamos víctimas de ataques o filtraciones por el solo hecho de trabajar remoto. Este temor es real, pero es todo un reto que se puede superar. $\mathrm{Al}$ adoptar teletrabajo, es necesario entonces que se tomen las medidas pertinentes para garantizar esa seguridad de la información (establecer políticas, instalar sistemas de apoyo) y por ende salvaguardar nuestros datos.

Fecha de recepción: 20 de julio de 2020 Fecha de aceptación: 14 de setiembre de 2020

Correspondencia: contacto@infocaribe.info yanna.stefanu@campus.eae.es 